\title{
Risk of venous thromboembolism in rheumatoid arthritis, and its association with disease activity: a nationwide cohort study from Sweden
}

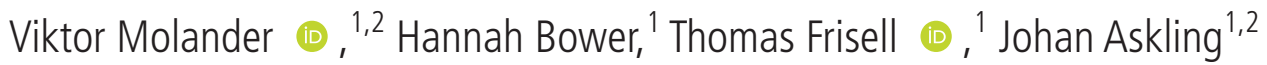

\section{Handling editor Josef S Smolen}

${ }^{1}$ Clinical Epidemiology Division, Karolinska Institutet, Stockholm, Sweden

${ }^{2}$ Rheumatology, Karolinska University Hospital, Stockholm, Sweden

\section{Correspondence to} Dr Viktor Molander, Clinical Epidemiology Division, Karolinska Institutet, Stockholm, Sweden; viktor.molander@ki.se

Received 24 June 2020 Revised 6 September 2020 Accepted 14 September 2020

Published Online First

8 October 2020
Check for updates

(C) Author(s) (or their employer(s)) 2021. No commercial re-use. See rights and permissions. Published by BMJ.

To cite: Molander $\mathrm{V}$ Bower $\mathrm{H}$, Frisell $\mathrm{T}$, et al. Ann Rheum Dis 2021:80:169-175

\section{ABSTRACT}

Objective To assess the incidence of venous thromboembolism (VTE) in rheumatoid arthritis (RA) relative to individuals without $R A$, and to investigate the relationship between aspects of clinical disease activity in RA and the risk of VTE.

Methods We conducted a nationwide register-based cohort study 2006 through 2018 using the Swedish Rheumatology Quality Register linked to other national patient registers to identify all patients with RA with at least one registered rheumatologist visit during the study period ( $n=46316$ patients, 322601 visits). The Disease Activity Score 28 erythrocyte sedimentation rate (ESR) (DAS28 ESR) and its components served as the exposure, and a VTE event within the year following the visit was the main outcome. We also included general population referents (1:5) matched on age, sex and residential area. Results Based on 2241 incident VTE events within 1 year of each included visit, and 5301 VTE events in the general population cohort, the risk ratio for VTE in RA was 1.88 (95\% Cl 1.65 to 2.15). Among patients with $\mathrm{RA}$, the risk (and risk ratio) increased with increasing RA disease activity, from $0.52 \%$ following visits in remission to $1.08 \%$ following visits with DAS28 ESR high disease activity, RR compared with remission $=2.03,95 \% \mathrm{Cl}$ 1.73 to 2.38. Compared with the general population, also patients with RA in DAS28 ESR remission were at elevated VTE risk.

Conclusions This study demonstrates a strong association between clinical RA disease activity measured by DAS28 ESR and the risk of VTE. RA disease activity can be used as an additional tool for VTE risk stratification in patients with RA.

\section{INTRODUCTION}

Venous thromboembolism (VTE), including deep vein thrombosis (DVT) and pulmonary embolism $(\mathrm{PE})$, are common medical concerns associated with significant morbidity and mortality. ${ }^{1}$ Established VTE risk factors include age, immobilisation, surgery, specific drugs and comorbid conditions such as malignancy, ischaemic heart disease, chronic obstructive pulmonary disease and hospitalised infection. $^{2}$

Several studies have demonstrated that patients with rheumatoid arthritis (RA) are, on average, at increased risk for VTE. ${ }^{3-10}$ By contrast, few if any studies have investigated the underlying reasons for this risk increase, let alone how it varies across patient subsets. Many established VTE risk factors occur more often in patients with RA. In vitro and

\section{Key messages}

What is already known about this subject?

- Patients with rheumatoid arthritis (RA) may be at increased risk of venous thromboembolism (VTE), but how any risk increase varies with RA disease activity is not known.

What does this study add?

- In this nationwide study, there was a close to doubled risk of VTE in patients with RA compared with the general population.

- We noted strong associations between measures of RA disease activity and risk of VTE events, for example, a twofold increase in risk from Disease Activity Score 28 (DAS28) remission to DAS28 high disease activity.

How might this impact on clinical practice or future developments?

- These results may be used as a basis for clinical VTE risk stratification in patients with RA.

in vivo studies have shown that aspects of inflammation might increase VTE risk by upregulation of procoagulatory factors and through endothelial damage. ${ }^{1112}$ Whether and how much clinical RA disease activity is linked to VTE risk remains to be understood. Such information would be important for a better understanding of the nature of the observed overall risk increase, and might constitute an important means for risk stratification in clinical practice, and in clinical trials.

The need for a better understanding of the association between the RA phenotype and VTE risk has radically increased with the recent safety signals arising from trials of Janus kinase inhibitors (JAKi). In 2019, and based on an increased number of VTE events in patients treated with the higher dose $(10 \mathrm{mg})$ in an ongoing postmarketing safety trial, the European Medicines Agency and the US Food and Drug Administration issued caution for VTE events in patients treated with tofacitinib. ${ }^{13} 14$ It remains unclear, however, if the purported VTE risk increase with JAKi is mainly explained by the drug itself, by the underlying disease, or by other factors.

The aims of this study were therefore to (1) investigate the relationship, if any, between clinical RA disease activity and the incidence of VTE, and 
(2) for contextualisation, to assess the incidence of VTE in RA patients relative to individuals without RA.

\section{METHODS}

\section{Study design}

We performed a nationwide cohort study of the association between clinical RA disease activity and VTE risk. We also compared VTE incidence in the RA population relative to matched general population referents.

\section{Setting}

Sweden has a population of around 10 million. Healthcare is publicly funded for all residents. Drug prescriptions are free of charge after an annual threshold of $€ 220$. Most patients with RA are treated by rheumatologists based at public hospitals.

\section{Data sources}

We identified a cohort of patients with RA from the Swedish Rheumatology Quality Register (SRQ), a large longitudinal clinical RA register operated by the Swedish Society for Rheumatology since the mid-1990s. SRQ contains longitudinal information on RA disease activity and treatment. Typically, at each outpatient visit to a rheumatologist, data are entered into SRQ by the rheumatologist, data on patient-reported outcome measures are entered by the patient. Cumulatively, SRQ holds information on around 52000 patients with RA. We linked this RA cohort to a series of other national and population-based Swedish registers: The Swedish National Patient Register, the Prescribed Drug Register, the Cause of Death Register and the Swedish Population Register. The National Patient Register contains information, including all registered International Classification of Diseases (ICD) codes, on hospital discharges from inpatient care since 1969, and visit data from non-primary outpatient care since 2001. Since 1987, the inpatient coverage is $>99 \% .{ }^{15}$ The Prescribed Drug Register contains information on all dispensations of prescribed drugs since 2005. The Cause of Death Register contains information about all deaths and causes (main and contributory ICD codes) since 1961. The Swedish Population Register contains information on residence and domicile, civil status and migration data for all inhabitants of Sweden. Individual-level data from these registers can be linked together using a unique personal identification number issued to all Swedish residents.

\section{Study population and exposure}

We identified all patients $\geq 18$ years of age with a rheumatologistbased diagnosis of RA, and who had at least one visit registered in the SRQ during 1 January 2006 to 31 December 2017 (46 316 patients with RA who contributed data from 322601 visits). For each visit, we obtained data on Disease Activity Score 28 ESR (hereafter DAS28) and its components (main outcome), Health Assessment Questionnaire (HAQ) and other clinical RA-variables, where available. We categorised each visit by its recorded DAS28 category; remission (0-2.6), low (2.7-3.2), intermediate (3.3-5.0) and high (>5.0) disease activity. For each unique patient, we randomly selected five referents from the Swedish Population Register $(n=215$ 843), individually matched by sex, year of birth and residential area. Online supplemental table S1 describes the creation of the datasets.

\section{Outcome}

Through linkage to the Patient Register, the Prescribed Drug Register and the Cause of Death Register we identified incident
VTE events occurring during the 365 days after each registered visit. One individual could thus contribute to more than one visit (and with more than one VTE event); each visit had its own baseline covariate status. In the main analysis, incident VTE was defined as a registration of any VTE diagnosis within the 365-day period in the Patient Register (inpatient and specialised outpatient care) or PE listed as underlying cause of death in the Cause of Death Register (online supplemental table S2 and online supplemental figure S1). We excluded visits for which the patient had a registered VTE event within the prior year, since VTE during this time was considered a prevalent VTE. Patients with a more distant history of VTE were included. If death (other than from PE) or emigration occurred during the 1-year follow-up that visit was excluded. For the general population referents, the 1-year follow-up period for assessment of incident VTE events started at the same date as the rheumatologist visit for their corresponding patient with RA.

\section{Statistical analyses}

We calculated the cumulative 1-year incidence for VTE in the RA population, by each DAS28 category, and in the general population referents. Risk ratios for the association between each DAS28 category and VTE risk, and for the RA population versus the general population referents, were calculated using log-binomial regression. We used robust cluster SEs to account for the correlated data structure in which one individual could contribute more than one visit. Models were adjusted for age at visit, via a restricted cubic spline with $3 \mathrm{df}$, sex and calendar year of visit (categorised 2006-2009, 2010-2013, 2014-2017). To test the robustness of our findings in relation to the definition of VTE, the length of the time window and the selection of rheumatologist visits, we performed a series of sensitivity analyses, as summarised in online supplemental table S2. We also performed multiple imputation (MI) using chained equations with $30 \mathrm{impu}$ tations to impute missing DAS28 category (17\% of all visits); multinomial regression was used for MI which included the VTE outcome, indicators for PE/DVT, age, sex and year. All analyses were performed using Stata V.16. ${ }^{16}$

\section{RESULTS}

Table 1 displays characteristics at each rheumatologist-visit for the entire RA population, overall and separately according to the DAS28 category at the visit (percentage missing for each variable presented in online supplemental table S3). Comparing patient characteristics by DAS28 category, we noted even distribution regarding RA treatment and socioeconomic characteristics, but a slightly increased prevalence of comorbidities at visits with high DAS28 disease activity (vs remission). Online supplemental table S3 displays characteristics at the first and last visit.

Table 2 presents the number of VTE events and cumulative incidences of VTE for the entire RA population, by each DAS28 category, and for their general population referents, overall and by VTE subtype, sex and age. In the RA population, 2241 visits in 1360 unique individuals were followed by a VTE within 1 year. Of these, 1408 were DVT events and 833 were PE events.

The overall cumulative 1 -year incidence of VTE was $0.71 \%$ in the RA population (ie, across all DAS28 categories) and $0.36 \%$ among their general population referents, corresponding to an adjusted risk ratio of VTE in RA of 1.88 (95\% CI 1.65 to 2.15). In both populations, the incidence of DVT was about twice as that of PE. The cumulative incidence of VTE was higher in males, and increased with increasing age. 
Table 1 Characteristics at each rheumatologist visit for entire study population and stratified by DAS28 category, in Swedish patients with RA registered in the Swedish Rheumatology Quality register from 2006 until 2017

\begin{tabular}{|c|c|c|c|c|c|}
\hline & \multirow[b]{2}{*}{ RA population } & \multicolumn{4}{|l|}{ DAS28 category } \\
\hline & & Remission & Low & Intermediate & High \\
\hline Observations (n) & 322601 & 97347 & 43756 & 94611 & 33217 \\
\hline Individuals (n) & 46316 & 29264 & 22637 & 31611 & 17385 \\
\hline Age at visit, median (IQR) & $63(52-71)$ & $62(50-70)$ & $64(54-72)$ & $63(53-71)$ & $63(53-71)$ \\
\hline Females (\%) & 74 & 67 & 75 & 78 & 78 \\
\hline RA duration, median (IQR) & $8.7(3.2-17.6)$ & $8.1(3.2-15.9)$ & $9.8(3.8-19.1)$ & $9.3(3.2-18.7)$ & $7.0(1.5-16.2)$ \\
\hline DAS28CRP, median (IQR) & $2.9(2.0-3.9)$ & $1.9(1.6-2.2)$ & $2.6(2.3-3.0)$ & $3.7(3.2-4.2)$ & $5.3(4.9-5.9)$ \\
\hline CRP, median (IQR) & $5.0(2.0-10.0)$ & $3.0(1.0-5.0)$ & $4.9(2.0-8.0)$ & $5.4(3.0-12.0)$ & $16.0(7.0-36.0)$ \\
\hline ESR, median (IQR) & $14.0(8.0-26.0)$ & $8.0(4.0-13.0)$ & $14.0(8.0-24.0)$ & $20.0(12.0-31.0)$ & $36.0(23.0-55.0)$ \\
\hline HAQ, median (IQR) & $0.8(0.3-1.3)$ & $0.3(0.0-0.8)$ & $0.8(0.3-1.1)$ & $1.0(0.6-1.4)$ & $1.4(1.0-1.9)$ \\
\hline Swollen joint count, median (IQR) & $1.0(0.0-3.0)$ & $0.0(0.0-0.0)$ & $0.0(0.0-2.0)$ & $2.0(1.0-4.0)$ & $8.0(5.0-11.0)$ \\
\hline Seronegative, unknown (\%) & 24 & 25 & 23 & 22 & 23 \\
\hline Smoker (\%) & 56 & 55 & 58 & 58 & 58 \\
\hline \multicolumn{6}{|l|}{ Comorbidities* } \\
\hline ACS (\%) & 2 & 2 & 3 & 3 & 3 \\
\hline Other cardiac disease (\%) & 26 & 22 & 27 & 28 & 30 \\
\hline VTE $(\%)$ & 1 & 1 & 1 & 1 & 2 \\
\hline Chronic kidney disease (\%) & 1 & 1 & 1 & 1 & 2 \\
\hline Cancer (in past 10 years) (\%) & 4 & 3 & 4 & 4 & 5 \\
\hline COPD $(\%)$ & 15 & 12 & 15 & 16 & 17 \\
\hline Diabetes (\%) & 9 & 7 & 9 & 10 & 12 \\
\hline Surgery $(\%) \dagger$ & 3 & 3 & 4 & 4 & 4 \\
\hline No previous biologics, median (IQR) & $1(1-3)$ & $1(1-2)$ & $1(1-3)$ & $1(1-3)$ & $1(1-3)$ \\
\hline NSAID/ASA (\%) & 59 & 54 & 58 & 63 & 69 \\
\hline Anticoagulant§ (\%) & 8 & 6 & 8 & 8 & 9 \\
\hline Oral oestrogen $(\%)$ & 14 & 13 & 15 & 15 & 14 \\
\hline \multicolumn{6}{|l|}{ Socioeconomic charcateristics } \\
\hline Married/cohabiting partner (\%) & 53 & 55 & 53 & 52 & 51 \\
\hline Disability pension in previous year (\%) & 2 & 2 & 2 & 2 & 2 \\
\hline Sick leave in previous year (\%) & 12 & 12 & 12 & 13 & 12 \\
\hline
\end{tabular}

*Registered within the last 5 years unless otherwise stated.

tSurgery (musculoskeletal, gynaecological, gastrointestinal or cardiovascular) within 90 days before visit.

FRA treatments: at time of visit. Other treatments: Registered within the last year.

$\S$ Collected anticoagulant drug from pharmacy within 1 year before VTE event.

ๆOral contraceptive w oestrogen or hormone replacement therapy.

ACS, acute coronary syndrome; b/tsDMARD, biological or targeted synthetic disease-modifying antirheumatic drug; COPD, chronic obstructive pulmonary disease; CRP, C reactive protein; DAS28,

Disease Activity Score 28; HAQ, Health Assessment Questionnaire; HAQ, Health Assessment Questionnaire; NSAID/ASA, non-steroidal anti-inflammatory drug/acetylsalicylic acid; RA, rheumatoid arthritis; TNFi, tumour necrosis factor inhabitor; VAS, visual analogue scale; VTE, venous thromboembolism.

Within the RA population, we noted a strong association between RA disease activity and the 1-year cumulative incidence of VTE, table 2 and figure 1 . The 1-year incidence of VTE events increased from $0.52 \%$ following visits in DAS28 remission, via $0.63 \%$ and $0.80 \%$ for low and moderate DAS 28 , respectively, to $1.08 \%$ following visit with a DAS28 above 5.1 , which corresponded to an adjusted risk ratio of VTE with high DAS28 RA disease activity (vs remission) of 2.03 (95\% CI 1.73 to 2.38).
When contrasting the DAS28 categories to the general population referents, the adjusted risk ratio of VTE was $1.34(95 \% \mathrm{CI}$ 1.13 to 1.58$)$ for patients in DAS28 remission, and $2.74(95 \% \mathrm{CI}$ 2.31 to 3.25 ) for those with high DAS28 (table 2).

Figure 2 displays the association between DAS28 and VTE stratified by VTE type (DVT vs PE), by personal history of VTE (yes vs no), by smoking status (smoker vs non-smoker) and by $\mathrm{C}$ reactive protein (CRP) level $(<5 \mathrm{vs} \geq 5)$ at the visit. For each 
Table 2 Cumulative incidence and risk ratio of VTE during 1-year follow-up after rheumatologist visit in Swedish patients with RA versus matched general population referents (1:5), and in DAS28 categories

\begin{tabular}{|c|c|c|c|c|c|c|}
\hline & \multicolumn{6}{|c|}{ No of VTE events (cumulative incidence, $\%$ ) } \\
\hline & \multicolumn{4}{|c|}{ Within RA, between DAS28 categories } & \multirow[b]{2}{*}{ Entire RA pop* } & \multirow[b]{2}{*}{ Gen pop } \\
\hline & Remission & Low & Intermediate & High & & \\
\hline Any & $496(0.52)$ & $271(0.63)$ & $743(0.80)$ & $350(1.08)$ & $2241(0.71)$ & $5301(0.36)$ \\
\hline DVT & $346(0.36)$ & $170(0.40)$ & $467(0.50)$ & $196(0.61)$ & $1408(0.45)$ & $3303(0.22)$ \\
\hline PE & $150(0.16)$ & $101(0.24)$ & $276(0.30)$ & $154(0.48)$ & $833(0.26)$ & $1998(0.14)$ \\
\hline Male & $188(0.60)$ & $71(0.66)$ & $190(0.94)$ & $78(1.10)$ & $664(0.80)$ & $1631(0.43)$ \\
\hline Female & $308(0.47)$ & $200(0.62)$ & $553(0.76)$ & $272(1.08)$ & $1577(0.68)$ & $3670(0.34)$ \\
\hline \multicolumn{7}{|l|}{ Age } \\
\hline $18-49$ & $37(0.16)$ & $23(0.30)$ & $54(0.31)$ & $24(0.39)$ & $174(0.27)$ & $419(0.13)$ \\
\hline $50-74$ & $332(0.54)$ & $174(0.62)$ & $450(0.74)$ & $215(1.01)$ & $1386(0.68)$ & $3541(0.37)$ \\
\hline 75- & $127(1.06)$ & $74(1.03)$ & $239(1.65)$ & $111(2.27)$ & $681(1.44)$ & $1341(0.71)$ \\
\hline Unadjusted & 1 (ref) & $1.22(1.05$ to 1.43$)$ & $1.56(1.37$ to 1.76$)$ & 2.10 (1.79 to 2.46$)$ & & \\
\hline \multirow[t]{3}{*}{ Adjusted } & 1 (ref) & $1.12(0.96$ to 1.31$)$ & $1.48(1.30$ to 1.68$)$ & 2.03 (1.73 to 2.38$)$ & & \\
\hline & \multicolumn{6}{|c|}{ RA population (including DAS28 categories) versus general population } \\
\hline & Remission & Low & Intermediate & High & Entire RA pop & Gen pop \\
\hline Unadjusted & $1.43(1.28$ to 1.59$)$ & 1.75 (1.52 to 2.01$)$ & 2.23 (2.02 to 2.45$)$ & 3.00 (2.62 to 3.43 ) & 1.96 (1.83 to 2.11$)$ & 1 (ref) \\
\hline Adjusted & 1.34 (1.13 to 1.58$)$ & 1.51 (1.25 to 1.82$)$ & 1.99 (1.72 to 2.31$)$ & 2.74 (2.31 to 3.25$)$ & 1.88 (1.65 to 2.15$)$ & 1 (ref) \\
\hline
\end{tabular}

Patients and referents followed from first visit registered after 2006 until 2018.

*Includes VTE events following a visit with missing DAS28 value ( $n=381,17 \%$ of all VTE events).

tRisk ratios adjusted for age, sex and calendar year.

DAS28, Disease Activity Score 28; DVT, deep vein thrombosis; PE, pulmonary embolism; RA, rheumatoid arthritis; VTE, venous thromboembolism.

of these analyses, we noted a pattern of increasing risk and risk ratios for VTE with increasing DAS28 similar to that of the main analysis. For instance, the risk ratio for PE was 3.06 (CI 2.36 to 3.97) for high DAS28 (vs remission), and the corresponding risk ratio for DVT was 1.59 (1.30-1.95). Importantly, although the risk ratios were largely similar across subgroups, the corresponding absolute 1-year risks varied across these patient subsets.

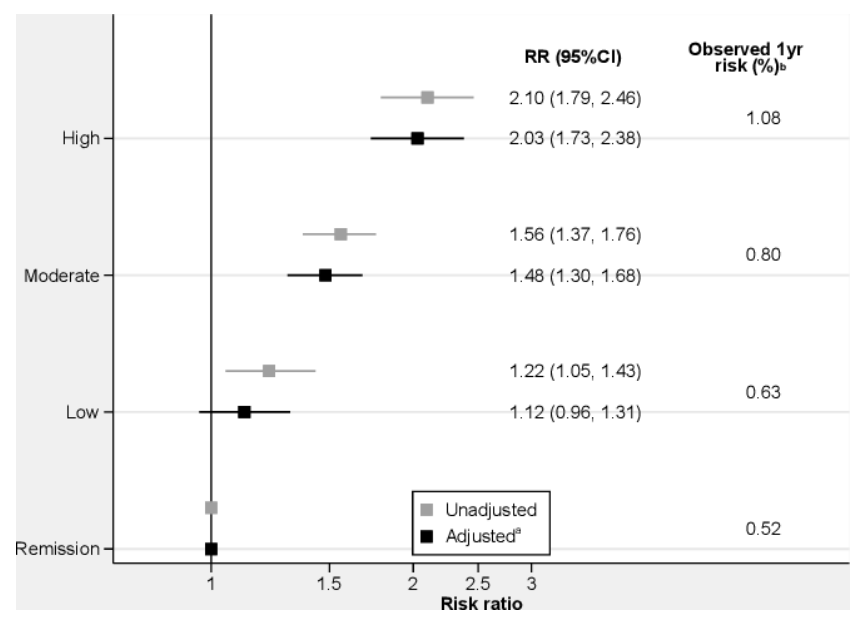

Figure 1 Risk ratios and absolute 1-year risks for the association between DAS28 and the risk of VTE within 1 year among Swedish

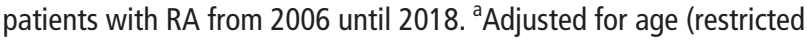
cubic spline), sex and calendar year of the visit year (categorised 20062009, 2010-2013, 2014-2017). ' Absolute 1-year risks are calculated from observed data. DAS28, Disease Activity Score 28; RA, rheumatoid arthritis; VTE, venous thromboembolism.
For instance, among individuals with high DAS28 disease activity, the 1year risk for VTE was $7.8 \%$ for individuals with a history of VTE compared with $1.0 \%$ for individuals without previous VTE. Risk ratios and 1-year risks for VTE were relatively similar across strata as defined by RA serostatus, RA disease duration, sex and oral glucocorticoids (online supplemental figure S2 and online supplemental table S5).

Table 3 presents the association between individual DAS28 components and VTE risk, and the association between HAQ and VTE risk. The pattern for each DAS28 component, as well as HAQ, was largely similar to that of the main analysis.

Online supplemental table S6 presents the predicted 1-year risk of VTE in RA, from log binomial models, by age and gender. The risks varied from $0.2 \%$ to $0.4 \%$ (remission vs high DAS28) in the 40 -year-old women, to the corresponding $1.1 \%-2.2 \%$ in the 80 -year-old men.

In sensitivity analyses that altered the VTE definition, the width of the time windows, changed various definitions of our study population, and using imputed DAS28 values, results were similar to our main analysis (online supplemental figures S3, S4 and tables S7, S8).

\section{DISCUSSION \\ Main findings}

In this large nationwide study, to our knowledge the first to specifically investigate the association between measures of RA disease activity and VTE risk, we found a strong association between RA disease activity as measured by DAS28, as well as by its components and by HAQ, and the risk of VTE during the following year. The increase in risk with high DAS28 was twice as high for PE as for DVT. We also noted that compared with the 


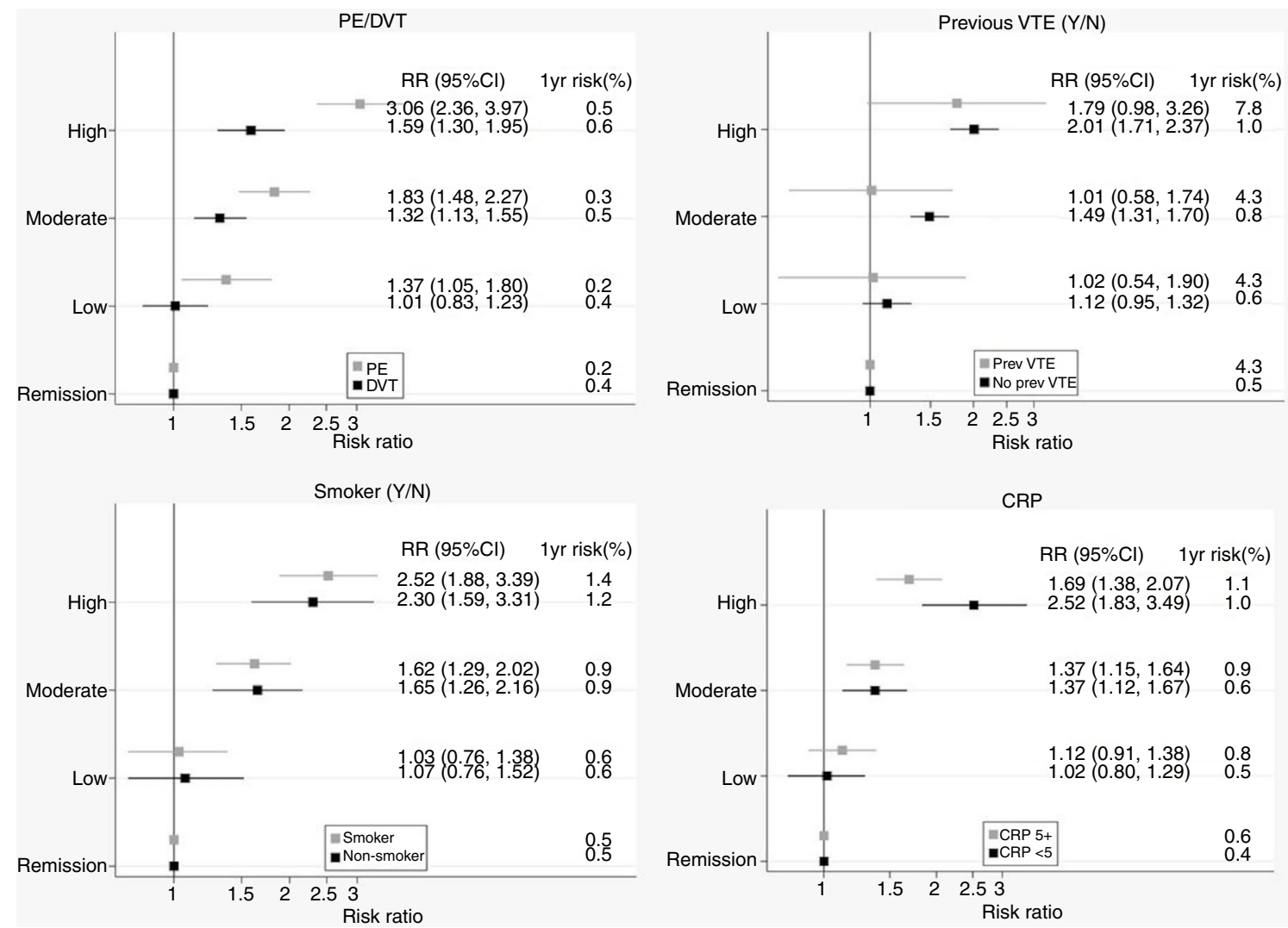

Figure 2 Risk ratios and absolute 1-year risks ${ }^{\text {a }}$ for the association between DAS28 and VTE by specified stratification within 1 year among Swedish patients with RA. ${ }^{a}$ Observed 1-year risk and adjusted risk ratios from log-binomial regression models adjusted for age (restricted cubic spline), sex and calendar year of the visit year (categorised 2006-2009, 2010-2013, 2014-2017). Absolute 1-year risks are calculated from observed data. CRP, C reactive protein; DAS28, Disease Activity Score 28; PE, pulmonary embolism; RA, rheumatoid arthritis; VTE, venous thromboembolism.

general population, even patients in DAS28 remission were at elevated VTE risk.

Few previous studies have investigated the relationship between characteristics in RA and VTE risk. One study on patients with RA $(n=253875)$ from a US claims database reported an increased incidence of inpatient VTE events in patients switching biologic or targeted synthetic disease-modifying antirheumatic drug (b/ tsDMARD) treatment compared with patients remaining on a first b/tsDMARD or only conventional synthetic DMARD. ${ }^{17}$ One might speculate that patients who switch b/tsDMARD on average have a higher disease activity than those who do not. If so, then the finding of an increased incidence of VTE among switchers of $b / t s D M A R D$ could be congruent with our findings, even if switching b/tsDMARD may occur for many other reasons than uncontrolled disease activity. Regarding RA disease activity and its role in coagulation, a cross-sectional study of 85 patients with RA reported an association between the rotational thromboelastometry (ROTEM) functional evaluation of the clotting cascade in whole blood, and DAS28. ${ }^{18}$ However, in that study, the clinical correlation between the ROTEM analyses and incidence of clinical VTE was not studied.

Our results including stratifications and sensitivity analyses indicated a remarkable consistency in the pattern of association between RA disease activity and VTE risk. The risk ratios for the association between DAS28 and VTE were almost identical for patients with and without a history of VTE, demonstrating that the association between DAS28 and VTE risk is not confined to the patients with this history. At the same time, the absolute risk differences were much higher in patients with a history of VTE ( $8 \%$ vs $1 \%$ risk with high DAS28), indicating that the clinical significance of high RA disease activity is much larger in this patient subset, and that VTE risk stratification is especially important in this group of patients. By contrast, when stratified by CRP ( $<5$ vs $\geq 5$ ), the associations between increasing DAS2 8 and VTE risk were similar, as were the absolute 1-year risks in highly active RA (1.0\% vs $1.1 \%)$, underscoring that the main result of this study is not necessarily driven by inflammation itself. Elevated CRP is known to cause pro-thrombotic activity and play a role in the pathogenesis of arterial thromboembolic events, although true causality with respect to VTE has not been established. ${ }^{19}$ We also noted an association between HAQ and VTE risk, underscoring the known association between functional status/ mobility and VTE risk. Our stratification on smoking (yes vs no) showed no difference in either RR or absolute 1-year risk, but the limited access to smoking data in our population should be considered.

Important to keep in mind, this study investigated VTE risk in a 12 (and 6, respectively) month window after the visit, and therefore, does not claim that the VTE risk is particularly high at any specific time point in this window. Also, we set out to investigate the association between indices of RA disease activity and VTE risk, not risks with individual DMARDs or treatment strategies.

In keeping with our main aim of studying the association between DAS28 and VTE, we adjusted for age, sex and calendar period but not for other risk factors. Our study population mainly comprised prevalent RA, therefore, at the time point of each visit it is not possible to fully distinguish comorbidities and other covariates that might be true confounders from such that are consequential to the RA disease and potential mediators of 


\begin{tabular}{|c|c|c|c|}
\hline & $\begin{array}{l}\text { No of VTE } \\
\text { events } \\
\text { (cumulative } \\
\text { incidence, \%) }\end{array}$ & $\begin{array}{l}\text { Risk ratio* } \\
(95 \% \mathrm{Cl}) \\
\text { Unadjusted }\end{array}$ & $\begin{array}{l}\text { Risk ratio } \\
(95 \% \mathrm{Cl}) \\
\text { Adjusted }\end{array}$ \\
\hline \multicolumn{4}{|c|}{ Swollen joint count } \\
\hline 0 & $811(0.59)$ & 1 (ref) & 1 (ref) \\
\hline $1-2$ & $532(0.72)$ & 1.24 (1.10 to 1.39$)$ & 1.27 (1.13 to 1.43$)$ \\
\hline $3-28$ & $817(0.87)$ & 1.49 (1.33 to 1.67$)$ & 1.55 (1.38 to 1.74$)$ \\
\hline \multicolumn{4}{|c|}{ Tender joint count } \\
\hline 0 & $772(0.61)$ & 1 (ref) & 1 (ref) \\
\hline $1-3$ & $688(0.74)$ & $1.22(1.10$ to 1.36$)$ & 1.29 (1.16 to 1.44$)$ \\
\hline $4-28$ & $699(0.82)$ & 1.35 (1.19 to 1.52$)$ & 1.52 (1.35 to 1.72$)$ \\
\hline \multicolumn{4}{|l|}{ ESR } \\
\hline $0-10$ & $589(0.55)$ & 1 (ref) & 1 (ref) \\
\hline $11-21$ & $595(0.70)$ & 1.28 (1.12 to 1.45$)$ & 1.11 (0.97 to 1.26$)$ \\
\hline $22-488$ & $833(0.88)$ & 1.61 (1.41 to 1.83$)$ & 1.24 (1.09 to 1.43$)$ \\
\hline \multicolumn{4}{|c|}{ Patient global health } \\
\hline $0-20$ & $564(0.55)$ & 1 (ref) & 1 (ref) \\
\hline $21-49$ & $609(0.65)$ & 1.17 (1.03 to 1.32$)$ & 1.12 (0.99 to 1.27$)$ \\
\hline $50-100$ & $896(0.92)$ & 1.66 (1.47 to 1.89$)$ & 1.65 (1.46 to 1.87$)$ \\
\hline \multicolumn{4}{|l|}{ HAQ } \\
\hline $0-0.38$ & $519(0.53)$ & 1 (ref) & 1 (ref) \\
\hline $0.39-1.00$ & $569(0.63)$ & 1.17 (1.01 to 1.35$)$ & 1.10 (0.95 to 1.27$)$ \\
\hline $1.01-3.00$ & $879(0.95)$ & 1.77 (1.55 to 2.04$)$ & 1.48 (1.29 to 1.71$)$ \\
\hline
\end{tabular}

*Risk ratios estimated from separate unadjusted and adjusted (age, sex and calendar year of the visit year) log-binomial regression models where each of the separate exposures were categorised according to their tertiles.

DAS28, Disease Activity Score 28; HAQ, Health Assessment Questionnaire; VTE, venous thromboembolism.

the very association under study. Our results, therefore, accurately reflect clinical risks and relative risks for VTE in RA and how these vary across RA disease activity and patient subsets, but do not directly inform on the relative importance of different components of this risk, for example, the relative contribution of different established VTE risk factors on this association. While directly applicable to clinical practice and amenable for use for clinical risk stratification, the results of our study, therefore, do not necessarily reflect any direct causality between each aspect of RA disease activity and VTE risk.

\section{Limitations}

Using a clinical register for identifying VTE events has benefits but could potentially result in underdiagnosis or overdiagnosis, and patients with RA are at risk for musculoskeletal conditions that could be misdiagnosed as DVTs. We, therefore, investigated a series of alternative definitions of VTE, including or not also anticoagulant treatment, and also considered DVT and PE events separately. Since the results demonstrated a pattern remarkably consistent with our main analysis, we find it unlikely that misclassification of VTEs had any significant effect on our results. Using DAS28 as a measure of disease activity is also a potential source of misclassification of true RA disease activity, since any concomitant condition causing elevated ESR, such as malignancies, will contribute to the DAS28 score. However, since the risk ratios for ESR and VTE risk were, if anything, lower than those for other individual DAS28 components, such misclassification of ESR is unlikely to be a source of significant bias in our study. We had somewhat limited data on smoking, and for certain other variables of interest, such as body mass index and immobility, information was not available.

\section{Strengths}

Using the SRQ to identify our RA cohort, we were able to include around $90 \%$ of all Swedish patients with RA treated by rheumatologists, which reduces the potential for selection bias and increases the generalisability of our findings. We linked these data to other nationwide registers based on prospectively recorded data of high internal validity and coverage for information on other variables, thereby reducing selection and information bias, and enabling comparisons both within RA and vs the general population. Our study demonstrated risk ratios of around 2.0 for VTE (DVT as well as PE) in RA compared with the general population. These results are similar to previous reports. For instance, in a previous study from our group, from 1997 to 2006 (ie, before the start of our study period) we noted HRs of around 2. ${ }^{3}$ A UK study of a prevalent RA population ( $\mathrm{n}=9589)$ from 1986 to 2010, reported RRs of around 2.2 for VTE, DVT and PE. ${ }^{8}$ This consistency further speaks to the generalisability of the results regarding the association between RA disease activity and VTE risk.

\section{CONCLUSION}

In conclusion, we found evidence of a strong association, with clinically relevant differences in absolute risks, between RA disease activity measured by DAS28 and the subsequent risk of VTE, which may be used for clinical risk stratification. Also, patients in remission are at increased risk vs the general population. The absolute risk increase with disease activity highlights the need for proper VTE risk assessment in patients with RA, especially for those with a history of VTE or other known risk factors. Our findings also suggest that patients with active RA, such as those typically recruited to phase III trials, are at particular elevated risk for VTE.

Correction notice This article has been corrected since it published Online First. Table 2 has been increased in size for clarity.

Acknowledgements We would like to thank all Swedish RA patients and rheumatologists for entering data into the Swedish Rheumatology Quality Register.

Contributors All authors participated in the design of the study. HB conducted the statistical analyses. VM, HB, TF and JA contributed to interpretation of the results. VM and JA contributed to the drafting of the manuscript. All authors contributed to the critical revision of the manuscript for important intellectual content. The study was supervised by JA.

Funding This study has received funding from Swedish Research Council, the Swedish Heart Lung Foundation, The Swedish Cancer Society, and the Karolinska Institutet Region Stockholm funds (ALF).

Disclaimer Funders had no impact on the design or interpretation of the study or its results.

Competing interests Karolinska Institutet, with $\mathrm{JA}$ as principal investigator, has or has had research agreements with Abbvie, Astra-Zeneca, BMS, Eli Lilly, MSD, Pfizer, Roche, Samsung Bioepis, Sanofi, and UCB, mainly in the context of safety monitoring of biologics via ARTIS/Swedish Biologics Register.

Patient and public involvement Patients and/or the public were involved in the design, or conduct, or reporting, or dissemination plans of this research. Refer to the Methods section for further details.

Patient consent for publication Not required.

Ethics approval Regional Ethics Committee, Stockholm, Sweden. 2015/1844$31 / 2$.

Provenance and peer review Not commissioned; externally peer reviewed.

Data availability statement Data may be obtained from a third party and are not publicly available. The study data forms part of a register linkage performed by Karolinska Institutet, and for which further sharing of the data is limited by legal restrictions. 
ORCID iDs

Viktor Molander http://orcid.org/0000-0003-0087-2565

Thomas Frisell http://orcid.org/0000-0002-5735-9626

\section{REFERENCES}

1 Heit JA, Silverstein MD, Mohr DN, et al. Predictors of survival after deep vein thrombosis and pulmonary embolism: a population-based, cohort study. Arch Intern Med 1999;159:445-53.

2 Cushman M. Epidemiology and risk factors for venous thrombosis. Semin Hematol 2007:44:62-9.

3 Holmqvist ME, Neovius M, Eriksson J, et al. Risk of venous thromboembolism in patients with rheumatoid arthritis and association with disease duration and hospitalization. JAMA 2012;308:1350-6.

$4 \mathrm{Kim}$ SC, Schneeweiss S, Liu J, et al. The risk of venous thromboembolism in patients with rheumatoid arthritis. Arthritis Care Res 2013;65:NA-7.

5 Chung W-S, Peng C-L, Lin C-L, et al. Rheumatoid arthritis increases the risk of deep vein thrombosis and pulmonary thromboembolism: a nationwide cohort study. Ann Rheum Dis 2014;73:1774-80.

6 Ungprasert P, Srivali N, Spanuchart I, et al. Risk of venous thromboembolism in patients with rheumatoid arthritis: a systematic review and meta-analysis. Clin Rheumatol 2014;33:297-304.

7 Bacani AK, Gabriel SE, Crowson CS, et al. Noncardiac vascular disease in rheumatoid arthritis: increase in venous thromboembolic events? Arthritis Rheum 2012;64:53-61.

8 Choi HK, Rho Y-H, Zhu Y, et al. The risk of pulmonary embolism and deep vein thrombosis in rheumatoid arthritis: a UK population-based outpatient cohort study. Ann Rheum Dis 2013;72:1182-7.
9 Ogdie A, Kay McGill N, Shin DB, et al. Risk of venous thromboembolism in patients with psoriatic arthritis, psoriasis and rheumatoid arthritis: a general population-based cohort study. Eur Heart J 2018;39:3608-14.

10 Zöller B, Li X, Sundquist J, et al. Risk of pulmonary embolism in patients with autoimmune disorders: a nationwide follow-up study from Sweden. Lancet 2012;379:244-9.

$11 \mathrm{Xu}$ J, Lupu F, Esmon CT. Inflammation, innate immunity and blood coagulation. Hamostaseologie 2010;30): :5-9. 5-6.

12 Borensztajn KS, von der Thüsen JH, Spek CA. The role of coagulation in chronic inflammatory disorders: a jack of all trades. Curr Pharm Des 2011;17:9-16.

13 EMA. Increased risk of blood clots in lungs and death with higher dose of Xeljanz (tofacitinib) for rheumatoid arthritis, 2019.

14 FDA. Safety trial finds risk of blood clots in the lungs and death with higher dose of tofacitinib (Xeljanz, Xeljanz XR) in rheumatoid arthritis patients. FDA to investigate 2019.

15 Ludvigsson JF, Andersson E, Ekbom A, et al. External review and validation of the Swedish national inpatient register. BMC Public Health 2011:11:450.

16 StataCorp. Stata statistical software: release 16. College Station, TX: StataCorp LLC, 2019

17 Liang H, Danwada R, Guo D, et al. Incidence of inpatient venous thromboembolism in treated patients with rheumatoid arthritis and the association with switching biologic or targeted synthetic disease-modifying antirheumatic drugs (DMARDs) in the realworld setting. RMD Open 2019;5:e001013.

18 Türk SM, Cansu Döndü Üsküdar, Teke Hava Üsküdar, et al. Can we predict thrombotic tendency in rheumatoid arthritis? A thromboelastographic analysis (with ROTEM). Clin Rheumatol 2018:37:2341-9.

19 Lippi G, Favaloro EJ, Montagnana M, et al. C-Reactive protein and venous thromboembolism: causal or casual association? Clin Chem Lab Med 2010;48:1693-701. 